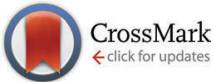

Cite this: J. Mater. Chem. B, 2016, 4, 4182

Received 5th April 2016,

Accepted 10th May 2016

DOI: $10.1039 / c 6 t b 00844 e$

www.rsc.org/MaterialsB

\section{Protein-aided formation of triangular silver nanoprisms with enhanced SERS performance $\dagger$}

\author{
Xi Geng, ${ }^{a}$ Weinan Leng, ${ }^{\text {bcd }}$ Nathan A. Carter, ${ }^{a}$ Peter J. Vikesland ${ }^{\text {bcd }}$ and \\ Tijana Z. Grove*a
}

In this work, we present a modified seed-mediated synthetic strategy for the growth of silver nanoprisms with low shape polydispersity, narrow size distribution and tailored plasmonic absorbance. During the seed nucleation step, consensus sequence tetratricopeptide repeat (CTPR) proteins are utilized as potent stabilizers to facilitate the formation of planar-twinned Ag seeds. Ag nanoprisms were produced in high yield in a growth solution containing ascorbic acid and CTPR-stabilized Ag seeds. From the time-course UV-Vis and transmission electron microscopy (TEM) studies, we postulate that the growth mechanism is the combination of facet selective lateral growth and thermodynamically driven Ostwald ripening. The resultant Ag nanotriangles (NTs) exhibit excellent surface enhanced Raman spectroscopy (SERS) performance. The enhancement factor (EF) measured for the 4-mercapto benzoic acid (4-MBA) reporter is estimated to be $3.37 \times 10^{5}$ in solution and $2.8 \times 10^{6}$ for the SERS substrate.

\section{Introduction}

Plasmonic metallic NPs such as Au and Ag NPs have received immense attention due to their tailored localized surface plasmon resonance (LSPR) band in the visible and near infrared (NIR) regions. $^{1,2}$ Since the electromagnetic field induced by the LSPR of NPs is strongly dependent upon the local dielectric environment, plasmonic NPs have been widely exploited for molecular sensing, bioimaging and therapeutic applications. ${ }^{3,4}$ Furthermore, the coupling of an electromagnetic field between adjacent NPs results in significant amplification of the spectroscopic signals allowing for the implementation of plasmonenhanced spectroscopic tools and other advanced optoelectronic devices. $^{5-7}$

Optical, electronic, and catalytic properties of NPs are determined by their composition, crystal structures, as well as their geometric features. ${ }^{1,8}$ The anisotropy at the nanoscale results in unprecedented physical and chemical properties. Therefore, over the past two decades, remarkable progress has been made

\footnotetext{
${ }^{a}$ Department of Chemistry, Virginia Polytechnic Institute and State University, Hahn Hall South, Blacksburg, VA 24060, USA. E-mail: tijana.grove@vt.edu; Tel: +1 540-231-7011

${ }^{b}$ Department of Civil and Environmental Engineering, Virginia Tech, Durham Hall, Blacksburg, VA 24061, USA

${ }^{c}$ Virginia Tech Institute of Critical Technology and Applied Science (ICTAS) Sustainable Nanotechnology Center (VTSuN), Virginia Tech, Kelly Hall, Blacksburg, VA 24061, USA

${ }^{d}$ Center for the Environmental Implications of Nanotechnology (CEINT), Duke University, Durham, NC 27708, USA

$\dagger$ Electronic supplementary information (ESI) available. See DOI: 10.1039/c6tb00844e
}

in the colloidal synthesis of a myriad of anisotropic NPs including nanowires, ${ }^{9,10}$ nanorods, ${ }^{11}$ nanoprisms ${ }^{12,13}$ and branched NPs. ${ }^{14-16}$ For example, the advent of $\mathrm{Ag}$ nanoprisms in early 2000 has sparked tremendous research interest in the subject of $2 \mathrm{D}$ platelike plasmonic nanostructures. ${ }^{12}$ Discrete dipole approximation (DDA) simulation and UV-Vis spectral investigations have unambiguously confirmed dual LSPR modes corresponding to the dipole and quadrupole. ${ }^{12,17}$ The in-plane dipole LSPR is conveniently regulated by the aspect ratio of nanoparticles (edge length/thickness). Moreover, electron energy-loss spectroscopy (EELS) mapping identified the plasmonic hot spots at the sharp tips of the nanoprisms. These plasmonic hot-spots imbibe nanoprisms with ultra-high chemical sensitivity that enables chemical and biological sensors for glucose, $\mathrm{Hg}^{+}$, and DNA. ${ }^{18-20}$ Furthermore, the massive field enhancement of these tips facilitates the fabrication of high-performance surface enhanced Raman spectroscopy (SERS) probes. ${ }^{5}$

Hitherto, a wide variety of colloidal routes have been developed to prepare well-defined $\mathrm{Ag}$ nanoprisms. ${ }^{21,22}$ Photo-induced approach through selective plasmon excitation triggers the transformation of $\mathrm{Ag}$ nanospheres into NTs with low polydispersity. 5,12,23,24 Despite high-precision control over the shape and size distribution of NPs, photochemical routes are time and energy-consuming processes that typically demand laser irradiation and can only proceed at low concentration thus limiting yields. By contrast, thermal approaches, either in aqueous or organic solutions, provide simple and rapid routes towards the high-yield production of Ag NTs. In particular, ligand-assisted chemical reduction methods are easier to perform and have been widely adopted. For example, polyvinyl pyrrolidone 
(PVP) and polyacrylamide (PA) with terminal hydroxyl groups serve as reductants and shape directing agents due to their excellent solubility and specific binding affinity to the metallic nanocrystals. $^{25,26}$ The fine shape control is realized by adjusting the ratio between the metal precursor and the reducing agent. ${ }^{26}$ Organic solvents such as dimethylformamide (DMF) and $n$-methyl-2pyrrolidone (NMP) have also been used for the formation of $\mathrm{Ag}$ NTs at elevated temperatures. ${ }^{27,28}$ In seminal studies by Mirkin and Yin's groups, $\mathrm{H}_{2} \mathrm{O}_{2}$ in synergy with citrate ions was used to selectively etch the facets of less-stable nanocrystals thereby promoting the nucleation of plate-like structures as well as the reconstruction of Ag NTs. ${ }^{29,30}$

Seed-mediated growth, proposed by Murphy et al. in their pioneering studies on gold nanorods and nanospheres with tunable size, ${ }^{11,31}$ has been successfully extended to prepare well-defined $\mathrm{Ag}$ nanoprisms with low shape and size polydispersity. ${ }^{13,32-36}$ Although multi-step protocols sacrifice the convenience of the traditional one-pot synthesis, exquisite control over the growth kinetics, and thus shape and size, is achieved by isolating the growth step from the initial nucleation of seeds. Notwithstanding that the underlying reasons by which the growth proceeds along one favourable orientation have not been indisputably addressed, seeded growth is believed to boost the symmetry breaking event during the course of shape evolution. ${ }^{37}$ Synthetic polymers such as PVP, sodium polystyrene sulfonate (PSSS), and polyvinyl alcohol (PVA) have been widely used as stabilizers and shape directing agents to aid the seededgrowth of $2 \mathrm{D} \mathrm{Ag}$ nanoprisms. ${ }^{38}$ Recently, $\mathrm{Ag}$ nanoprisms with a large lateral dimension $(\sim 5 \mu \mathrm{m})$ and a high aspect ratio $(\sim 400)$ have been realized by means of successive epitaxial deposition over multiple cycles. ${ }^{39,40}$

Biopolymer-assisted approaches have recently opened up the potential for the green-synthesis of anisotropic NPs under ambient conditions. ${ }^{41-43}$ For instance, single crystalline Au nanoprisms have been prepared using plant extracts such as lemongrass, ${ }^{44}$ and brown seaweed. ${ }^{45}$ Additionally, the so-called shape directing proteins, albeit of unknown sequence and structure, were isolated from the algal extract to produce Au nanoplates with distinctive triangular or hexagonal shapes. ${ }^{42}$ However, research so far has been focused on the one-pot biomimetic methods, which typically generate gold nanoprisms with rather large size and high shape polydispersity. Only a few attempts have been made on the bioassisted seeded growth of shape-selective noble NPs with improved morphological yield. ${ }^{46,47}$

Previously, our group has demonstrated that the morphology and optical properties of noble metal NPs could be modulated by the concentration and the structure of the CTPR proteins. ${ }^{48}$ CTPR is a de novo protein sequence based on the tertratricopeptide repeat family. ${ }^{49}$ The CTPR protein and repeat proteins in general are composed of a repeating structural motif. In the CTPR protein that motif is the helix-turn-helix motif with 34 amino acids in length. If all repeats have an identical sequence, as is the case for CTPR, the protein can be treated as a simple homopolymeric molecule, where the monomer is a single repeat. ${ }^{50}$ Extending the number of CTPR protein repeats results in a superhelical structure, where eight repeats $(n=8)$ comprise one full turn of

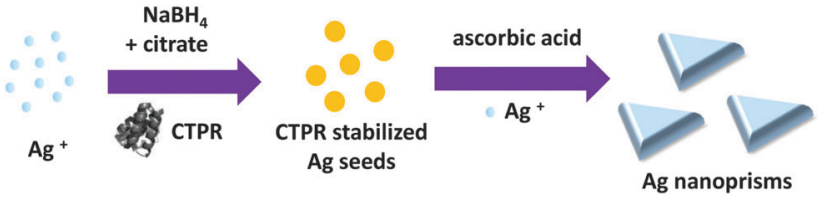

Scheme 1 Schematic illustration of synthetic procedures for triangular Ag nanoprisms.

the superhelix. ${ }^{51,52}$ In this work, we use a CTPR protein consisting of three repeats, which is the smallest stable molecule. ${ }^{51}$ The sequence of the CTPR protein and the circular dichroism (CD) spectrum indicative of the helical structure are shown in the ESI, $\dagger$ Fig. S1. The ${ }^{1} \mathrm{H}^{-15} \mathrm{~N}$ HSQC NMR and fluorescence quenching studies have been carried out in our lab to interrogate the interaction between the CTPR protein and noble metals. ${ }^{53}$ Binding of $\mathrm{Au}(\mathrm{III})$ to CTPR is driven by the overall electrostatics, with tryptophan, tyrosine and asparagine side chains coordinating the metal ion. Guided by that knowledge, we hypothesized that CTPR will similarly interact with silver ions and thus exert control over the kinetics, crystalline structure and preferential growth of silver NPs.

In the present study, we developed a modified protocol for seed-mediated synthesis of 2D anisotropic $\mathrm{Ag}$ nanoprisms (Scheme 1). Herein, engineered repeat proteins, CTPR proteins, were used to prepare well-dispersed $\mathrm{Ag}$ seeds with inherent twinning defects. These spherical nuclei then direct the lateral growth of well-defined Ag nanoprisms with tunable size and optical properties. Time-course UV-Vis and TEM studies provided insights into the mechanism of NP evolution, whilst the roles of the protein have also been elucidated. The as-prepared Ag nanoprisms exhibited remarkable SERS performance both in solution and on the solid substrate.

\section{Experimental section}

\subsection{Chemicals and reagents}

Silver nitrate (>99.5\%) was purchased from Fluka. Lysozyme was obtained from (Calbiochem) EMD Millipore Corporation. All other chemical reagents were acquired from Sigma-Aldrich and Fisher Chemicals and were used as received without further purification. Deionized water (18 $\mathrm{M} \Omega \mathrm{cm}^{-1}$, Millipore Milli-Q) was exclusively used for preparing all aqueous solutions and for all rinsing procedures.

\subsection{Expression and purification of CTPR proteins}

CTPR proteins were synthesized through recombinant bacterial expression technology as described previously (Fig. S1, ESI $\dagger)^{54,55}$ Synthetic genes for the desired protein in PPROEx vector, coding for $\mathrm{N}$-terminal (His) $)_{6}$ tag and ampicillin resistance, were transformed into $E$. coli BL21(DE3) cells and cultured overnight at $37^{\circ} \mathrm{C}$ on agar plates. A single colony was selected and incubated overnight in $50 \mathrm{ml}$ Luria-Bertani (LB) media containing $100 \mu \mathrm{g} \mathrm{ml}^{-1}$ ampicillin. $10 \mathrm{ml}$ of overnight cultures were then dispersed into $1 \mathrm{l}$ of fresh LB media supplemented with $100 \mu \mathrm{g} \mathrm{m}{ }^{-1}$ ampicillin. The cells were grown in an incubator-shaker $(250 \mathrm{rpm})$ at $37^{\circ} \mathrm{C}$ until the optical 
density $\left(\mathrm{OD}_{600}\right)$ reached 0.8. Expression of CTPR3 and CTPR3 with terminal cysteine (CTPR3-cys) was induced with $1 \mathrm{mM}$ isopropyl $\beta$-D-thiogalactoside (IPTG) followed by $5 \mathrm{~h}$ expression at $37{ }^{\circ} \mathrm{C}$, whereas CTPR6 and 18 were expressed at $18{ }^{\circ} \mathrm{C}$ for $16 \mathrm{~h}$ in an analogous manner. The cells were harvested by centrifugation at $5000 \mathrm{rpm}$ for $20 \mathrm{~min}$ and pellets were frozen at $-80{ }^{\circ} \mathrm{C}$ until purification.

The cell pellets were re-suspended in lysis buffer consisting of $50 \mathrm{mM}$ Tris, $300 \mathrm{mM}$ sodium chloride and $0.1 \mathrm{wt} \%$ Tween 20 . After 2 min of sonication at $30 \%$ power using a microtip and the Mison sonicator, lysed cells were centrifuged at $16000 \mathrm{rpm}$ for $30 \mathrm{~min}$ and protein supernatant was purified using a standard Ni-NTA affinity purification protocol. The N-terminal hexa-histidine tag was then cleaved from the CTPR proteins using TEV protease. The collected proteins were further purified by Akta Prime Plus size exclusion chromatography using a Superdex 75 16/600 or 200 16/600 Prep Grade column in $150 \mathrm{mM}$ sodium chloride and $50 \mathrm{mM}$ sodium phosphate buffer at $\mathrm{pH} 8$ with a flow rate of $0.5 \mathrm{ml} \mathrm{min}^{-1}$. As a final step, the aqueous solutions of proteins were dialyzed against $10 \mathrm{mM}$ phosphate buffer three times at $4{ }^{\circ} \mathrm{C}$ using a dialysis cassette with a molecular weight cutoff of $3.5 \mathrm{k}$ or $10 \mathrm{k} \mathrm{Da} .{ }^{55}$

\subsection{Preparation of CTPR-stabilized $\mathrm{Ag}$ seeds and $\mathrm{Ag}$ prisms}

Silver seeds were prepared by adding $5 \mathrm{ml}$ of $0.5 \mathrm{mM} \mathrm{AgNO}$ at the rate of $2 \mathrm{ml} \mathrm{min}^{-1}$ using a syringe pump (KD Scientific) under vigorous agitation to the aqueous solutions of trisodium citrate $(5 \mathrm{ml}, 2.5 \mathrm{mM}), \mathrm{NaBH}_{4}(0.3 \mathrm{ml}, 10 \mathrm{mM})$ and CTPR proteins (0.125 $\left.\mathrm{ml}, 0.1-2 \mathrm{mg} \mathrm{ml}^{-1}\right)$ in a glass vial. In the ensuing step, a fixed amount of CTPR-stabilized Ag seeds (10-200 $\mu \mathrm{l})$ was added to $5 \mathrm{ml}$ of $0.15 \mathrm{mM}$ ascorbic acid aqueous solution, followed by the dropwise addition of $3 \mathrm{ml}$ of $0.5 \mathrm{mM} \mathrm{AgNO}_{3}$ at the rate of $1 \mathrm{ml} \mathrm{m^{-1 }}{ }^{-1}$ under stirring. A series of $\mathrm{Ag}$ seeds and $\mathrm{Ag}$ NPs were prepared in this manner under ambient conditions by adjusting the concentration of both reactants and capping agents. BSA, lysozyme and CTPR proteins with different terminal functionalities and varied lengths (number of repeats) were employed as stabilizers and potential shape-directing agents (Fig. S1, ESI $\dagger$ ). The as-prepared $\mathrm{Ag}$ colloidal solution was centrifuged at 10000 rpm for $20 \mathrm{~min}$ and the resultant pellets were rinsed with DI water to remove the residue stabilizer and reducing agent. After several purification cycles, the Ag NTs were re-dispersed in DI water for later SERS measurement.

\subsection{Instrumentation}

TEM and high resolution TEM (HR-TEM) analyses were performed on a Philips EM420 at an accelerating voltage of $120 \mathrm{kV}$ and on a JEOL 2100 at $200 \mathrm{kV}$, respectively. TEM samples were prepared by applying $7 \mu \mathrm{l}$ of a sample solution on 300 mesh ultrathin carbon-coated $\mathrm{Cu}$ grids (EM Science), followed by drying overnight before observation. The thickness, as well as the size of Ag prisms, was measured from TEM images using Image $\mathrm{J}$. The morphology of Ag NPs is also characterized using a ZEISS 1550VP field emission scanning electron microscope (FESEM). The UV-Vis spectra of Ag NPs were monitored using an Agilent Cary 100 Bio UV-Vis spectrophotometer. All UV-Vis measurements were conducted in a $1 \mathrm{~cm}$ path length PMMA cuvette at room temperature. SERS spectra were collected using a WITec alpha 500R using a $785 \mathrm{~nm}$ excitation source. Briefly, $10 \mu \mathrm{l}$ of $10 \mathrm{mM}$ 4MBA solutions were added to $0.99 \mathrm{ml}$ of purified Ag colloidal solutions, followed by incubation on a benchtop shaker for $12 \mathrm{~h}$. The liquid samples were injected into a quartz cuvette (path length $=2 \mathrm{~mm}$ ) for SERS analysis. The self-assembled Ag NT SERS substrate was fabricated by drop casting purified Ag NT colloidal solution onto the Si wafer and then dried under ambient conditions. $5 \mu \mathrm{l}$ of $10 \mathrm{mM} 4 \mathrm{MBA}$ solution was subsequently transferred onto the SERS substrate. Backscattered light was collected using $100 \times$ objectives at integration times of $1 \mathrm{~s}$. The signal was dispersed using a 1200 grooves per $\mathrm{mm}$ grating and the dispersed light was collected using a Peltier-cooled charge-coupled device (CCD). All SERS spectra reported for the cuvettes or substrates are the average of 100 spectra collected over the identical focal area. ${ }^{6}$

\section{Results and discussion}

Seed-mediated synthesis of NPs is a versatile and robust method that decouples the growth stage from the initial nucleation thereby allowing for precise control over the size and shape of NPs. ${ }^{37,56}$ In the presence of seed nanocrystals, the deposition of metal atoms onto the pre-existing seeds is more favorable than homogenous nucleation due to the lower activation energy. With the aid of a surfactant, the NP evolution is dictated by the crystal structure of seeds as well as by the surfactant templating effect.

\subsection{Morphological characterization of CTPR-stabilized $\mathrm{Ag}$ seeds and $\mathrm{Ag}$ nanoprisms}

In the seed-nucleation step, spherical Ag NPs with the particle size ranging from 3-5 $\mathrm{nm}$ were produced in the presence of sodium citrate and CTPR3 protein, where 3 stands for three tandem repeats of the 34 amino acid sequence (Fig. 1). Additionally, a large number of $\mathrm{Ag}$ nanoclusters $(<2 \mathrm{~nm})$ were formed through rapid reduction of $\mathrm{AgNO}_{3}$ by $\mathrm{NaBH}_{4}$ (Fig. 1a). Golden yellow colloidal solution of CTPR3-stabilized Ag NPs has the extinction peak at $408 \mathrm{~nm}$. The distinctive twinning boundaries, which are regarded as one of the key prerequisites for the subsequent epitaxial growth of 2D planar nanoprisms, have been observed in HR-TEM images of Ag seeds (Fig. 1a inset). ${ }^{57}$ The as-prepared $\mathrm{Ag}$ seeds were kept in the dark until further use to circumvent interference such as homogeneous nucleation arising from the residual $\mathrm{NaBH}_{4}$. In the subsequent step, fast seed addition and dropwise $\mathrm{AgNO}_{3}$ injection into the reaction mixture enabled the rapid growth of high-quality anisotropic nanoprisms (Fig. 1b). ${ }^{13}$

Single crystalline structure of $\mathrm{Ag}$ prisms with the lattice spacing of $0.144 \mathrm{~nm}$ was confirmed by HR-TEM and the selected area diffraction (SAED) pattern indexed as (220), whilst the flat top face was ascertained by the weak SAED diffraction spots corresponding to the forbidden 1/3(422) specific for planar $\mathrm{Ag}$ or Au NTs (Fig. 1b inset). ${ }^{12}$ The average length of NTs is approximately $102.8 \pm 18.9 \mathrm{~nm}$ as illustrated in the representative TEM and SEM images of Ag NTs (Fig. 1c-e). The thickness of Ag NTs is estimated 

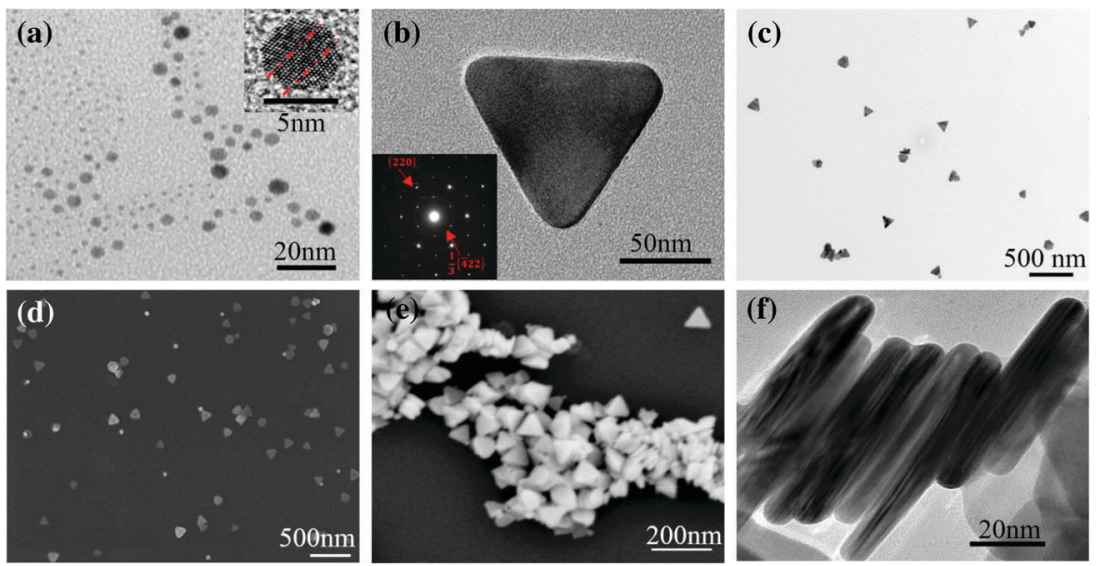

Fig. 1 (a) TEM image of CTPR3-Ag seeds (inset: HR-TEM image of a twinning nanocrystal), (b) HR-TEM image and selective area diffraction (SAED) pattern of Ag NTs, (c) TEM image and (d) SEM image of Ag NTs prepared using $20 \mu \mathrm{l}$ of CTPR3-Ag seeds. The thickness of Ag nanoprisms was determined from SEM (e) and TEM (f) images.

to be $8.8 \pm 2.0 \mathrm{~nm}$ from the images of vertically stacked NTs (Fig. 1e and f). The synthetic route is robust and reproducible - over $85 \%$ of the NPs are NTs or partially truncated NTs before any purification process. The absence of CTPR3 during the seed growth step results in a poor yield of Ag NTs under the same experimental conditions.

\subsection{Mechanistic investigation on the formation of Ag NTs}

Effect of citrate. We further investigated if citrate can be omitted from the seed-growth step and if CTPR3 solely could be used to adequately regulate the growth of Ag seeds. The LSPR intensity of Ag nanospheres increased by $20 \%$ in the absence of citrate but is accompanied by a broadening of the full width at the half maximum (FWHM) of the plasmon absorption peak, indicative of higher polydispersity (Fig. S2a, ESI $\dagger$ ). However, NPs prepared with these seeds lack dipolar in-plane LSPR absorbance in the NIR region indicating that these seeds are inefficient in activating the formation of Ag prisms (Fig. S2b, ESI $\dagger$ ). In fact, NPs with a variety of shapes including spheres, nanorods and nanoprisms are produced under these conditions (Fig. S2c, ESI†). Our observation is in good agreement with the earlier literature reporting that the citrate anions are critical for the transformation of spherical Ag NPs to Ag NTs. ${ }^{58}$ Both CTPR and citrate are thus indispensable for the high-yield production of Ag NTs through a seed-mediated process. Since only very low quantities of citrate or CTPR3 are present during the seededgrowth step, face-selective growth of Ag NTs is primarily driven by the innate planar twinning defects of $\mathrm{Ag}$ seeds rather than the strong absorbance of capping agents on the (111) facets of the $\mathrm{Ag}$ NTs. Moreover, we have also carried out a control experiment in which $23 \mu \mathrm{g} \mathrm{ml}^{-1}$ CTPR was added during the growth step instead of the seed nucleation step. The CTPR present in this step dramatically reduced the reaction kinetics leading to a poor yield of Ag nanoparticles that were primarily nanospheres.

Effect of ascorbic acid. Since the shape-control in the synthesis of anisotropic Ag NPs is typically achieved by adjusting the ratio of ascorbic acid (AA) to metal precursors, $\mathrm{Ag}$ crystal growth was performed under the conditions of varied concentrations of AA. The $\lambda_{\max }$ of LSPR red-shifted slightly to $c a .850 \mathrm{~nm}$ when the ratio of AA to $\mathrm{Ag}$ was doubled (Fig. S3a, ESI $\dagger$ ). This result can be rationalized by the extended edge length arising from the accelerated lateral deposition of $\mathrm{Ag}(0)$ atoms onto the edge of Ag NTs with high surface energy. With the further increase in the $[\mathrm{AA}] /\left[\mathrm{Ag}^{+}\right]$ratio and thus the reaction rate, the selfnucleation process could not be efficiently inhibited, ${ }^{56}$ resulting in thermodynamically favourable products such as cuboctahedra or quasi-globular Ag NPs enclosed by a mixture of both (111) and (100) facets. Excess of AA is also considered to induce undesirable defects onto the Ag NTs such as dislocations and stacking faults leading to the deformation of NTs and gradual damping of the LSPR intensity (Fig. S3a and b, ESI $\dagger$ ). According to the UV-Vis profile and TEM images, the ratio of $[\mathrm{AA}] /\left[\mathrm{Ag}^{+}\right]$in the range from 0.5 to 1 is needed for the high-yield of well-defined nanoprisms with intact outlines.

Morphology development. During the course of the reaction outlined in Fig. 2, the transformation of ultrafine spherical Ag seeds to $2 \mathrm{D}$ nanoprisms with rounded edges and eventually to Ag NTs was revealed by the gradual color change from pale yellow to ruby red, then blue and finally purple. To elucidate the underlying growth mechanism, parallel experiments were performed in which a fixed volume of $\mathrm{Ag}^{+}$precursors was added to the solution of CTPR3-Ag seeds followed by a rapid injection of $0.5 \mathrm{ml}$ of $25 \mathrm{mM}$ citrate to suppress continuous growth, i.e. the quenching reaction. As shown in Fig. 2, the initial injection of $0.05 \mathrm{ml}$ of $\mathrm{AgNO}_{3}$ was rapidly reduced by the excess of AA promoting the enlargement of spherical $\mathrm{Ag}$ seeds and leading to the formation of small $2 \mathrm{D} \mathrm{Ag}$ NPs with a disk-like or triangular contour. Addition of $0.1 \mathrm{ml} \mathrm{of} \mathrm{Ag}^{+}$led to the lateral growth of the NPs on the relatively high-energy facets such as (110) and (100). Concurrently, a large amount of small Ag nanoclusters (stage II) is produced. Intriguingly, only triangles or disks with the size around $40 \mathrm{~nm}$ are observed after addition of $0.2 \mathrm{ml}$ of $\mathrm{AgNO}_{3}$, whereas quasi-spherical $\mathrm{Ag} \mathrm{NPs}$ and a majority of nanoclusters almost disappeared (stage III). This is likely due to the Ostwald ripening, i.e. small nanoclusters or nanospheres composed of a large number of surface atoms are 


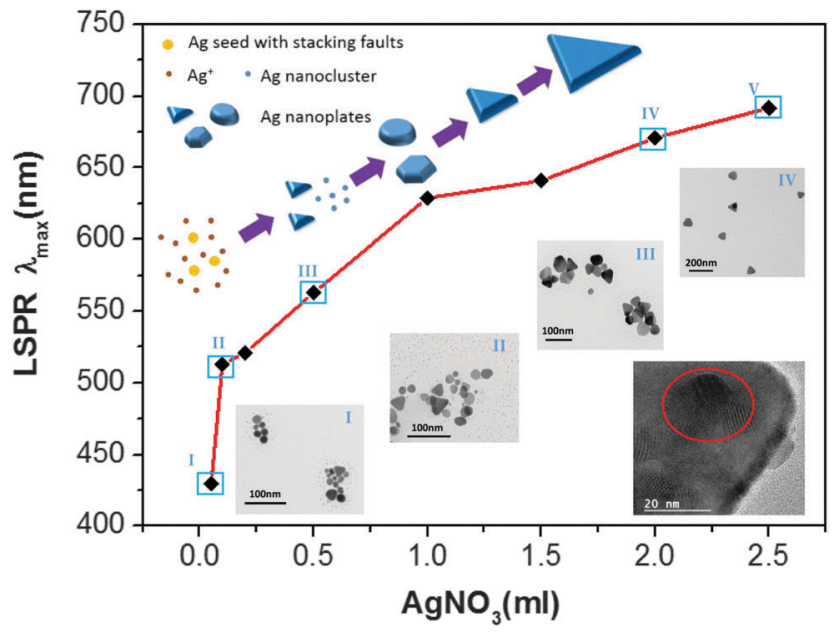

Fig. 2 Mechanism of Ag NTs formation. The LSPR wavelength and TEM images corresponding to different injection volumes of $0.5 \mathrm{mM} \mathrm{AgNO}_{3}$. Inset: HR-TEM images of protrusions fused onto the nanoprisms.

etched by $\mathrm{O}_{2}$ provided during the vigorous agitation, followed by the condensation on the adjacent larger NPs, preferentially at the higher edge sites. ${ }^{59}$ As clearly seen in the Fig. 2 inset, some protrusions and fused small hemispheres are identified on the top and at the edge of the Ag NTs. Further increase in the precursor amount allows for the continuous lateral deposition of $\mathrm{Ag}(0)$ onto the $\mathrm{Ag}$ prisms in a face-selective manner and the round disks are converted to NTs (stage IV). The structural transition from small triangles to round disks and finally back to large triangular nanoprisms has been previously observed by Yin et al. ${ }^{60}$ The individual edges of small triangles formed at the early stage are composed of one hcp facet sandwiched by one (111) and one (100) fcc facet of varied thickness. ${ }^{35}$ The preferential deposition of $\mathrm{Ag}$ on (100) sub-facets leads to the formation of intermediates such as truncated hexagonal nanoplates or round disks possessing a mixture of (100) and (111) bounded edges. The shape evolution ceases when all the (100) facets disappear, accompanied by the formation of intact triangles. The observed LSPR red shift of the $\lambda_{\text {max }}-408 \mathrm{~nm} \rightarrow 430 \mathrm{~nm} \rightarrow 513 \mathrm{~nm} \rightarrow 623 \mathrm{~nm}-$ is consistent with the proposed mechanism and shape evolution from the small $\mathrm{Ag}$ seeds to bigger $\mathrm{Ag}$ nanospheres to small $2 \mathrm{D}$ nanoprisms and finally to larger NTs (Fig. 2 and Fig. S4, ESI $\dagger$ ). We surmise that the following two mechanisms act in concert: (1) atom-by-atom deposition of newly reduced $\mathrm{Ag}(0)$ onto the highenergy edges of preformed nanoprisms; and (2) dissolution and merging of tiny spherical NPs and nanoclusters onto the anisotropic 2D NTs. ${ }^{61}$

Effect of CTPR3 sequence on the Ag seeds and the Ag NTs. To further explore the distinct role of CTPR3, control experiments were performed in which commercially available Bovine Serum Albumin (BSA) and lysozyme were utilized as stabilizers in the synthesis of Ag seeds. Additionally, we also prepared Ag seeds in the absence of CTPR3 as shown in Fig. S5a (ESI $\dagger$ ). Well-dispersed $\mathrm{Ag}$ seeds were prepared in the presence of BSA, even though the FWHM of UV-Vis absorbance is broader than for seeds prepared with CTPR3 indicating the higher size polydispersity. In sharp contrast, severe agglomeration of lysozyme-Ag NPs occurs resulting in a broad hump in the UV-Vis spectra (Fig. S5a, ESI + ).

Next, we proceeded with NT synthesis. $20 \mu \mathrm{l}$ of as-prepared Ag seeds stabilized with either CTPR3, BSA, or lysozyme were used in the reaction as described. Whereas the colloidal solution synthesized using CTPR3-Ag seeds exhibits strong absorbance peaks in the NIR region (793 nm), ascribed to the characteristic in-plane dipolar LSPR band of Ag NPs (Fig. S5b, ESI $\dagger$ ), no apparent LSPR bands were identified in the NIR region of colloidal solutions prepared using other seeds. This observation is consistent with TEM images where NPs prepared with CTPR3 seeds are well-defined NTs with a narrow size distribution (Fig. S5c, ESI $\dagger$ ) but a variety of shapes are present when using other Ag seeds (Fig. S5d-f, ESI $\dagger$ ).

The observed effect of protein used in AgNP synthesis can be rationalized by a closer inspection of protein physicochemical properties. CTPR3 and BSA have similar pI values, 4.5 and 4.7, respectively, but BSA has almost five times larger molecular weight. Additionally, CTPR3 does not contain any thiols whereas BSA does. On the other hand CTPR3 and lysozyme are similar in size, but lysozyme has a pI of 11.35. Thus we suggest that neither thiols nor positive charge is favourable for the growth of NTs. The cysteine or disulphide moieties - present in both BSA and lysozyme - have strong binding affinities for Ag and potentially inhibit both the growth of a low-energy (111) top facet and the lateral growth of high energy (110) and (100) facets on the edges. Concurrently, the positive charges and sulfhydryl and disulphide functionalities may also lead to the undesirable assembly of NPs as shown in Fig. S5d and e (ESI $\dagger$ ).

To date, a series of amino acid residues have been extensively exploited to modify the surface properties or regulate the growth of metal NPs. ${ }^{62-65}$ In particular, the terminal polyhistidine and cysteine tag is often deliberately engineered into the protein or peptide for the controlled assembly or patterning of NPs. ${ }^{6-70}$ To better understand the tolerance of the described synthetic strategy to modifications of CTPR3, we performed Ag NT synthesis using either CTPR3 with a N-terminal polyhistidine tag or CTPR3 with an engineered C-terminal cysteine. Although the UV-Vis spectra of colloidal solution containing Ag seeds are identical in all cases (Fig. S6a, ESI $\dagger$ ), the intensity of LSPR peaks is noticeably lower for Ag NTs prepared via CTPR3-cys-Ag seeds in contrast to their counterparts (Fig. S6b, $\mathrm{ESI} \dagger$ ). Ag NPs with large size and shape polydispersity are generated in the reaction with CTPR3-cys-Ag seeds similar to that observed for BSA-Ag seeds (Fig. S6c, ESI $\dagger$ ). Conversely, reaction with poly-histidine CTPR3-Ag seeds resulted in the formation of fully-developed triangles with a small degree of truncations and snips (Fig. S6d, ESI $\dagger$ ).

Although the specific interactions between CTPR3 and Ag NPs are not yet known, we hypothesize that abundant aromatic residues, i.e. tyrosine and tryptophan in the CTPR sequence play a role analogous to polystyrene sulfonate or aromatic surfactants, such as benzyldimethylhexadecylammonium chloride, ${ }^{10}$ in promoting the growth of high-quality Ag seeds. Rather than through facet-selective adsorption or the so-called surfactant templating mechanism, the precise shape control is attained 
primarily due to the nature of twinned crystal seeds setting stage for the plate-like structures during the growth stage. The CTPR sequence also contains negatively charged aspartate residues that have been shown to be advantageous to the high yield synthesis of nanoplates. ${ }^{65}$ Furthermore, the electrostatic repulsion due to surface charges precludes the NP fusion process during the seed formation and the initial NT growth step, ${ }^{24}$ thus improving the colloidal stability and reducing the polydispersity. In contrast to our previous findings that protein shape may exert an appreciable impact on the growth and morphology of gold NPs, ${ }^{48}$ the morphology as well as the optical properties of Ag NTs are independent of the size and shape of the CTPR protein. (Fig. S6a, b and e)

Effect of CTPR3 concentration. Since CTPR3 has been confirmed to improve the quality of the Ag seeds as well as the overall morphological yield of the Ag NTs, we explored the optimal CTPR3 concentration. With the increase in the CTPR3 concentration from $2.3 \mu \mathrm{g} \mathrm{ml}^{-1}$ to $15.4 \mu \mathrm{g} \mathrm{ml}^{-1}$, the LSPR band of CTPR3-bound Ag seeds was slightly narrowed (Fig. 3a). A further increase in CTPR3 concentration caused a dramatic attenuation of the LSPR intensity, potentially as a consequence of complexation between $\mathrm{Ag}^{+}$and CTPR leading to the slow reaction. $\mathrm{Ag}$ seeds prepared in the presence of lower concentrations of CTPR3 (2.3-23 $\left.\mu \mathrm{g} \mathrm{ml}^{-1}\right)$ are indeed more favourable for subsequent NT formation (Fig. $3 \mathrm{~b}$ and d).

When Ag NPs are prepared using CTPR3-Ag seeds with a low concentration of CTPR3 $\left(2.3 \mu \mathrm{g} \mathrm{ml}^{-1}\right)$, not only Ag triangles but tetrahedra, octahedra, and pentatwins bound to (111) facets are concurrently produced (Fig. 3c). The larger shape polydispersity is probably due to the poor quality of seeds containing defects like multi-fold twining structures. Contrary to that, an excessive amount of CTPR3 $\left(>46 \mu \mathrm{g} \mathrm{ml}^{-1}\right)$ may significantly slow down the nucleation rate of $\mathrm{Ag}$ seeds and cause a detrimental effect on the follow-up synthesis of Ag NTs. Indeed, small-sized quasiglobular NPs and NTs with irregular contours are observed in the TEM images (Fig. 3e). This observation implies that the suppression of growth is no longer limited to (111) on the top faces but also takes place on the reactive concave and convex facets at edges. The appearance of a vast number of small nanoclusters around the NPs also indicates that the hypothesized Ostwald ripening is to some extent halted under such circumstances. Therefore, CTPR3 concentration ranging from 15.4 to $23 \mu \mathrm{g} \mathrm{ml}^{-1}$ is proposed as the optimal concentration during the seed nucleation step.

A small shift (408 nm $\rightarrow 415 \mathrm{~nm}$ ) and a minor loss in LSPR intensity (11\%) for $\mathrm{Ag}$ seeds occurred after storage in the dark for two weeks (Fig. S2a, ESI $\dagger$ ). Although LSPR of NTs undergoes a slight blue shift $(793 \mathrm{~nm} \rightarrow 765 \mathrm{~nm})$, the yield and the morphology of the Ag NTs resemble those of Ag NTs prepared using fresh Ag seeds (Fig. S2b, ESI $\dagger$ and Fig. 2d). Overall, both CTPR3-functionalized Ag seeds and the resultant Ag NTs exhibit excellent colloidal stability and structural integrity under ambient conditions since no significant precipitation or peak broadening occurs during the storage. The long-term colloidal stability could be further improved by storing NPs in oxygen and a halide free environment and by applying an extra capping agent.

\subsection{Tailoring the morphology and plasmon bands of $\mathbf{A g}$ nanoprisms through adjusting the amount of CTPR3-Ag seeds}

One striking advantage of the seed-mediated protocol is that the LSPR could be conveniently tuned to span a broad spectral range, from the visible to the NIR region (500-900 $\mathrm{nm}$ ), through the variation of the quantities of $\mathrm{Ag}$ seeds used during the growth step. ${ }^{35}$ Precise control over the LSPR is attainable when less seeds $(<100 \mu \mathrm{l})$ are used. Otherwise, a bimodal absorbance profile is observed, attributed to the competitive growth between isotropic nanospheres and facet-selected NTs in the presence of a large number of nuclei (Fig. 4g). The LSPR features are strongly dependent upon the morphological features of the Ag NTs including their edge length, thickness as well as the sharpness of the vertices. ${ }^{21}$ As the total number of seeds increased, the mean particle size declined steadily and the resultant Ag NPs underwent a progressive shape transition from triangular nanoprisms to round disks (Fig. 4a-e). The remarkable change in the shape
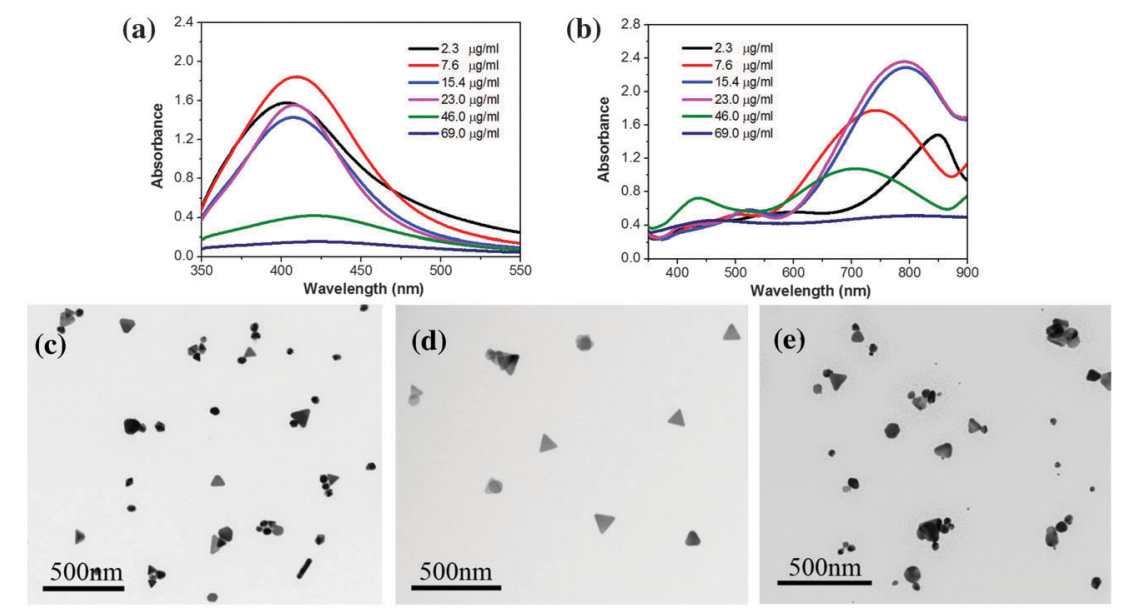

Fig. 3 UV-Vis spectra of (a) Ag seeds prepared with increasing CTPR3 concentration and (b) Ag NPs prepared using the corresponding CTPR-Ag seeds; (c-e) TEM images of Ag NPs prepared using $20 \mu \mathrm{l}$ of CTPR3-Ag seeds $\left(2.3 \mu \mathrm{g} \mathrm{ml}^{-1} ; 15.4 \mu \mathrm{g} \mathrm{ml}^{-1}\right.$; and $46 \mu \mathrm{g} \mathrm{ml}{ }^{-1}$ protein). 

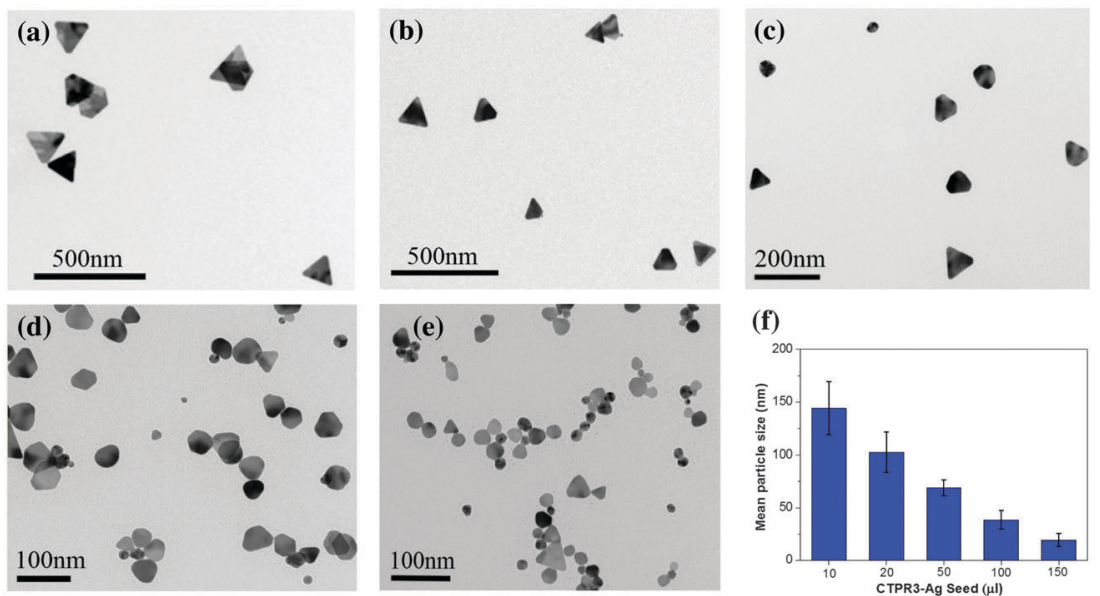

(g)

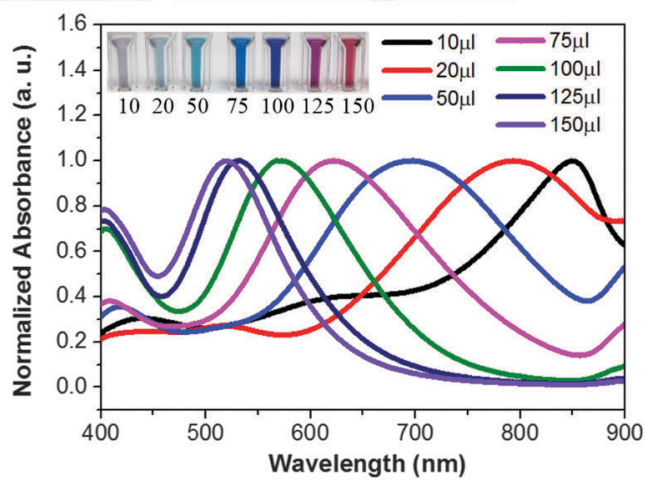

Fig. 4 TEM images of selected samples prepared using (a) 10, (b) 20, (c) 50, (d) 100, and (e) $150 \mu$ l CTPR3-Ag seed, (f) mean particle size vs. volume of seeds, (g) UV-Vis spectra and the photographic images of the collodial Ag NTs solutions obtained using different volumes of seeds.

and size consequently bring about the dramatic blue shift of LSPR absorbance as shown in Fig. $4 \mathrm{~g}$.

\subsection{SERS performance and EF calculation}

The SERS performance of the Ag NTs was investigated using 4-MBA as reporter molecule. As illustrated in Fig. 5a, 4MBA tagged Ag NTs exhibited two intense peaks at 1078 and $1590 \mathrm{~cm}^{-1}$, which could be assigned to the characteristic aromatic ringbreathing mode. ${ }^{71}$ The enhancement factor $(\mathrm{EF})$ was estimated using eqn (1) for the SERS probe in solution and using eqn (2) for the SERS substrate:

$$
\begin{gathered}
\mathrm{EF}=\left(I_{\text {SERS }} / I_{\text {Sol }}\right) \times\left(C_{\text {Sol }} / C_{\text {SERS }}\right) \\
\mathrm{EF}=\left(I_{\text {SERS }} / I_{\text {Bulk }}\right) \times\left(N_{\text {Bulk }} / N_{\text {SERS }}\right)
\end{gathered}
$$

where $I_{\text {SERS }}$ and $I_{\text {Sol }}$ are the peak intensities at $1078 \mathrm{~cm}^{-1}$ for the 4MBA@Ag solution and 0.5 M 4MBA solution in $1 \mathrm{M} \mathrm{NaOH}$, and $C_{\text {SERS }}\left(N_{\text {SERS }}\right)$ and $C_{\text {Sol }}\left(N_{\text {Sol }}\right)$ are the concentration (number) of the SERS sample and ordinary 4MBA. Assuming that a monolayer of 4MBA molecules with a footprint of $0.54 \mathrm{~nm}^{2}$ is adsorbed onto the $\mathrm{Ag}$ prisms in solution, the $\mathrm{EF}$ value is estimated to be $3.37 \times 10^{5}$. This value could be slightly underestimated due to the incomplete surface coverage (see the ESI $\dagger$ for detailed calculation). ${ }^{5}$ Alternatively, we can also evaluate the EF of the SERS substrate by making comparison between the SERS signal of $4 \mathrm{MBA}$ on the Ag NT substrate and the Raman signal of the 4MBA bulk sample on Si wafer (Fig. 5b). By using identical measurement parameters including the accumulation time and the laser focal volume, the EF value is estimated to be $2.8 \times 10^{6}$. Previous study has shown that 63 hot spots per million SERS sites contribute $24 \%$ of the overall signal intensity. ${ }^{72}$ As compared with other SERS surfaces, ${ }^{73,74}$ the Ag NT-based SERS substrate may exhibit the following features (highlighted by blue circles in Fig. 5c): (a) a few bowtie-like structures are observed in both Fig. 5c and Fig. S7 (ESI $\dagger$ ) in which two Ag NTs are facing tip to tip with a few nanometer gap. This well-oriented bowtie patterning has been previously shown to make a great enhancement on Raman signals. ${ }^{75}$ (b) Additionally, the close packing of adjacent $\mathrm{Ag}$ NTs may also provide a number of potential hot spots that may significantly amplify the electromagnetic field thereby obtaining a higher EF value. ${ }^{5}$ Thus we hypothesized that the near-field coupling between adjacent NPs with sharp tips will generate a large number of hot spots further augmenting the EF value. Undoubtedly, the as-prepared $\mathrm{Ag}$ prisms with well-defined morphologies and sharp vertices can serve as highly active SERS-tags or substrate materials for the sensing and detection of a trace amount of analytes.

\section{Conclusion}

In summary, CTPR proteins have been explored for the bioenabled synthesis of anisotropic Ag nanoparticles. We present 
(a)

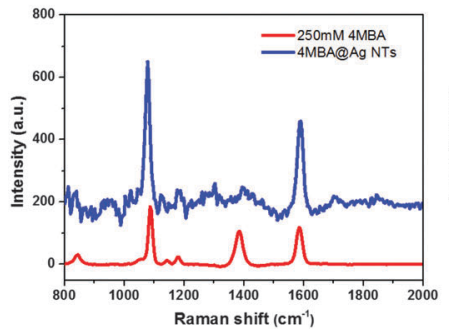

(b)

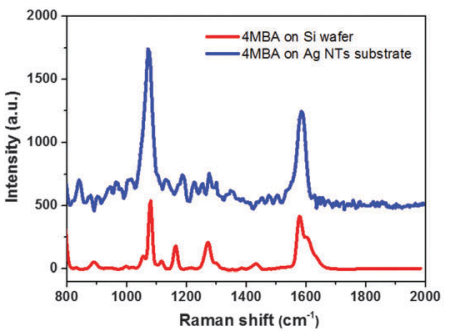

(c)

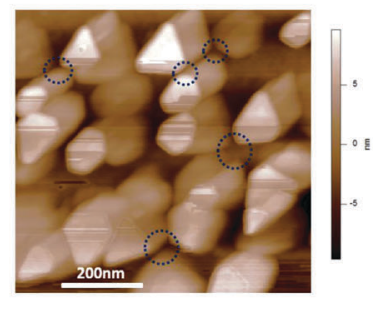

Fig. 5 Raman spectra of (a) 250 mM 4MBA in a solution containing 1 M NaOH and 4MBA-taged Ag NTs colloidal solution. (b) 4MBA on the Si wafer and on the Ag NTs SERS substrate. (c) Representative AFM images of the SERS substrate prepared by drop casting Ag NTs onto the silicon wafer.

a facile and rapid synthetic strategy for the preparation of $\mathrm{Ag}$ nanoprisms under ambient conditions based on a CTPRassisted seed-growth. Through the systematic study of various experimental parameters, we found that the presence of CTPR3 is pivotal for the production of well-dispersed planar-twinned seeds necessary for the high-yield growth of $\mathrm{Ag}$ nanotriangles with low polydispersity. The well-defined Ag nanoprisms are fabricated using $c a .23 \mu \mathrm{g} \mathrm{ml}^{-1}$ CTPR3 during the seed nucleation step while the AA to $\mathrm{AgNO}_{3}$ ratio ranging from 0.5-1 is preferred during the NT growth step. Furthermore, we provide insights into the mechanism of Ag NT generation. The facet selective epitaxial growth and thermodynamically driven Ostwald ripening act concurrently to yield Ag NTs of $c a .100 \mathrm{~nm}$ edge-length and $8 \mathrm{~nm}$ thickness. Plasmon absorbance ranging from the visible to the NIR region can be tailored by adjusting the amount of CTPR3stabilized seeds in the reaction. These Ag NTs exhibit excellent SERS performance. The EF of a 4-MBA reporter molecule is estimated to be $3.37 \times 10^{5}$ for the Ag NT based SERS probe in solution and $2.8 \times 10^{6}$ for the solid substrate.

\section{Conflicts of interest}

The authors declare no competing financial interest.

\section{Acknowledgements}

The authors would like to thank Dr Guoliang Liu and Clayton Scruggs for insightful comments, suggestions, and discussion on this research work. The authors acknowledge ICTAS Nanoscale Characterization and Fabrication Lab (NCFL) for the use of AFM, TEM, and SEM. This work was in part supported by the JFC ICTAS grant number 119106 to TZG.

\section{References}

1 I. Pastoriza-Santos and L. M. Liz-Marzan, J. Mater. Chem., 2008, 18, 1724-1737.

2 Q. Zhang, Y. N. Tan, J. Xie and J. Y. Lee, Plasmonics, 2008, 4, 9-22.

3 E. C. Dreaden, A. M. Alkilany, X. Huang, C. J. Murphy and M. A. El-Sayed, Chem. Soc. Rev., 2012, 41, 2740-2779.

4 O. Tokel, F. Inci and U. Demirci, Chem. Rev., 2014, 114, 5728-5752.
5 B. Xue, D. Wang, J. Zuo, X. Kong, Y. Zhang, X. Liu, L. Tu, Y. Chang, C. Li, F. Wu, Q. Zeng, H. Zhao, H. Zhao and H. Zhang, Nanoscale, 2015, 7, 8048-8057.

6 W. Leng and P. J. Vikesland, Anal. Chem., 2013, 85, 1342-1349.

7 L. A. Lane, X. Qian and S. Nie, Chem. Rev., 2015, 115, 10489-10529.

8 S. E. Lohse, N. D. Burrows, L. Scarabelli, L. M. Liz-Marzán and C. J. Murphy, Chem. Mater., 2014, 26, 34-43.

9 Y. Sun, B. Mayers, T. Herricks and Y. Xia, Nano Lett., 2003, 3, 955-960.

10 Z. Qian and S.-J. Park, Chem. Mater., 2014, 26, 6172-6177.

11 A. Gole and C. J. Murphy, Chem. Mater., 2004, 16, 3633-3640.

12 R. Jin, Y. Cao, C. A. Mirkin, K. L. Kelly, G. C. Schatz and J. G. Zheng, Science, 2001, 294, 1901-1903.

13 L. Scarabelli, M. Coronado-Puchau, J. J. Giner-Casares, J. Langer and L. M. Liz-Marzán, ACS Nano, 2014, 8, 5833-5842.

14 J. Xie, J. Y. Lee and D. I. C. Wang, Chem. Mater., 2007, 19, 2823-2830.

15 C.-H. Kuo and M. H. Huang, Langmuir, 2005, 21, 2012-2016.

16 S. Saverot, X. Geng, W. Leng, P. J. Vikesland, T. Z. Grove and L. R. Bickford, RSC Adv., 2016, 6, 29669-29673.

17 J. E. Millstone, S. Park, K. L. Shuford, L. Qin, G. C. Schatz and C. A. Mirkin, J. Am. Chem. Soc., 2005, 127, 5312-5313.

18 Y. Xia, J. Ye, K. Tan, J. Wang and G. Yang, Anal. Chem., 2013, 85, 6241-6247.

19 L. Chen, X. Fu, W. Lu and L. Chen, ACS Appl. Mater. Interfaces, 2013, 5, 284-290.

20 X. Yang, Y. Yu and Z. Gao, ACS Nano, 2014, 8, 4902-4907.

21 J. E. Millstone, S. J. Hurst, G. S. Métraux, J. I. Cutler and C. A. Mirkin, Small, 2009, 5, 646-664.

22 H. Yu, Q. Zhang, H. Liu, M. Dahl, J. B. Joo, N. Li, L. Wang and Y. Yin, ACS Nano, 2014, 8, 10252-10261.

23 R. Jin, Y. Charles Cao, E. Hao, G. S. Metraux, G. C. Schatz and C. A. Mirkin, Nature, 2003, 425, 487-490.

24 C. Xue and C. A. Mirkin, Angew. Chem., Int. Ed., 2007, 46, 2036-2038.

25 Y. Xiong, A. R. Siekkinen, J. Wang, Y. Yin, M. J. Kim and Y. Xia, J. Mater. Chem., 2007, 17, 2600-2602.

26 Y. Xiong, I. Washio, J. Chen, H. Cai, Z.-Y. Li and Y. Xia, Langmuir, 2006, 22, 8563-8570.

27 I. Pastoriza-Santos and L. M. Liz-Marzán, Nano Lett., 2002, 2, 903-905. 
28 M. H. Kim, D. K. Yoon and S. H. Im, RSC Adv., 2015, 5, 14266-14272.

29 G. S. M. Metraux and A. Chad, Adv. Mater., 2005, 27, 2685.

30 Q. Zhang, N. Li, J. Goebl, Z. Lu and Y. Yin, J. Am. Chem. Soc., 2011, 133, 18931-18939.

31 N. R. Jana, L. Gearheart and C. J. Murphy, Langmuir, 2001, 17, 6782-6786.

32 T. K. Sau and C. J. Murphy, J. Am. Chem. Soc., 2004, 126, 8648-8649.

33 S. Chen and D. L. Carroll, Nano Lett., 2002, 2, 1003-1007.

34 S. Chen, Z. Fan and D. L. Carroll, J. Phys. Chem. B, 2002, 106, 10777-10781.

35 D. Aherne, D. M. Ledwith, M. Gara and J. M. Kelly, Adv. Funct. Mater., 2008, 18, 2005-2016.

36 N. Li, Q. Zhang, S. Quinlivan, J. Goebl, Y. Gan and Y. Yin, ChemPhysChem, 2012, 13, 2526-2530.

37 W. Niu, L. Zhang and G. Xu, Nanoscale, 2013, 5, 3172-3181. 38 A. Le Beulze, E. Duguet, S. Mornet, J. Majimel, M. TréguerDelapierre, S. Ravaine, I. Florea and O. Ersen, Langmuir, 2014, 30, 1424-1434.

39 Q. Zhang, Y. Hu, S. Guo, J. Goebl and Y. Yin, Nano Lett., 2010, 10, 5037-5042.

40 J. Zeng, X. Xia, M. Rycenga, P. Henneghan, Q. Li and Y. Xia, Angew. Chem., Int. Ed., 2011, 50, 244-249.

41 L. Yu, I. A. Banerjee and H. Matsui, J. Am. Chem. Soc., 2003, 125, 14837-14840.

42 J. Xie, J. Y. Lee, D. I. C. Wang and Y. P. Ting, Small, 2007, 3, 672-682.

43 R. R. Naik, S. J. Stringer, G. Agarwal, S. E. Jones and M. O. Stone, Nat. Mater., 2002, 1, 169-172.

44 S. S. Shankar, A. Rai, B. Ankamwar, A. Singh, A. Ahmad and M. Sastry, Nat. Mater., 2004, 3, 482-488.

45 B. Liu, J. Xie, J. Y. Lee, Y. P. Ting and J. P. Chen, J. Phys. Chem. B, 2005, 109, 15256-15263.

46 F. Jiang and Y.-L. Hsieh, Biomacromolecules, 2014, 15, 3608-3616.

47 V. Singh, P. Khullar, P. N. Dave, G. Kaur and M. S. Bakshi, ACS Sustainable Chem. Eng., 2013, 1, 1417-1431.

48 X. Geng and T. Z. Grove, RSC Adv., 2015, 5, 2062-2069.

49 E. R. G. Main, K. Stott, S. E. Jackson and L. Regan, Proc. Natl. Acad. Sci. U. S. A., 2005, 102, 5721-5726.

50 N. A. Carter and T. Z. Grove, Biomacromolecules, 2015, 16, 706-714.

51 T. Kajander, A. L. Cortajarena, S. Mochrie and L. Regan, Acta Crystallogr., Sect. D: Biol. Crystallogr., 2007, 63, 800-811.

52 A. L. Cortajarena, J. Wang and L. Regan, FEBS J., 2010, 277, 1058-1066.
53 K. L. Roth, X. Geng and T. Z. Grove, J. Phys. Chem. C, 2016, DOI: $10.1021 /$ acs.jpcc.6b02569.

54 T. Kajander, A. L. Cortajarena, E. R. G. Main, S. G. J. Mochrie and L. Regan, J. Am. Chem. Soc., 2005, 127, 10188-10190.

55 A. L. Cortajarena, F. Yi and L. Regan, ACS Chem. Biol., 2008, 3, 161-166.

56 Y. Xia, X. Xia and H.-C. Peng, J. Am. Chem. Soc., 2015, 137, 7947-7966.

57 M. L. Personick and C. A. Mirkin, J. Am. Chem. Soc., 2013, 135, 18238-18247.

58 Y. Sun and Y. Xia, Adv. Mater., 2003, 15, 695-699.

59 L. Wang, X. Wu, X. Li, L. Wang, M. Pei and X. Tao, Chem. Commun., 2010, 46, 8422-8423.

60 J. Goebl, Q. Zhang, L. He and Y. Yin, Angew. Chem., Int. Ed., 2012, 51, 552-555.

61 B. Radha and G. U. Kulkarni, Cryst. Growth Des., 2011, 11, 320-327. 62 A. Swami, A. Kumar, M. D'Costa, R. Pasricha and M. Sastry, J. Mater. Chem., 2004, 14, 2696-2702.

63 B. Roy, S. Mukherjee, N. Mukherjee, P. Chowdhury and S. P. Sinha Babu, RSC Adv., 2014, 4, 34487-34499.

64 M. Annadhasan, T. Muthukumarasamyvel, V. R. Sankar Babu and N. Rajendiran, ACS Sustainable Chem. Eng., 2014, 2, 887-896.

65 Y. Shao, Y. Jin and S. Dong, Chem. Commun., 2004, 1104-1105, DOI: 10.1039/B315732F.

66 T. Ueno, T. Koshiyama, T. Tsuruga, T. Goto, S. Kanamaru, F. Arisaka and Y. Watanabe, Angew. Chem., Int. Ed., 2006, 45, 4508-4512.

67 N. Hom, K. R. Mehta, T. Chou, A. B. Foraker, F. M. Brodsky, K. Kirshenbaum and J. K. Montclare, J. Mater. Chem., 2012, 22, 23335-23339.

68 J. Xie, J. Y. Lee and D. I. C. Wang, J. Phys. Chem. C, 2007, 111, 10226-10232.

69 R. A. McMillan, J. Howard, N. J. Zaluzec, H. K. Kagawa, R. Mogul, Y.-F. Li, C. D. Paavola and J. D. Trent, J. Am. Chem. Soc., 2005, 127, 2800-2801.

70 Y. Bhattacharjee and A. Chakraborty, ACS Sustainable Chem. Eng., 2014, 2, 2149-2154.

71 J. Li, J. Zhou, T. Jiang, B. Wang, M. Gu, L. Petti and P. Mormile, Phys. Chem. Chem. Phys., 2014, 16, 25601-25608.

72 Y. Fang, N.-H. Seong and D. D. Dlott, Science, 2008, 321, 388-392.

73 X. Hong, D. Wang and Y. Li, Chem. Commun., 2011, 47, 9909-9911.

74 L.-f. Zhang and C.-y. Zhang, Nanoscale, 2013, 5, 6074-6080.

75 H. Zhou, F. Yu, C. F. Guo, Z. Wang, Y. Lan, G. Wang, Z. Fang, Y. Liu, S. Chen, L. Sun and Z. Ren, Nanoscale, 2015, 7, 9153-9157. 